\title{
Pathological significance of ophthalmic foreign bodies
}

\begin{abstract}
Foreign bodies (FB) in eye and adnexa are important in ophthalmology particularly in traumatic cases. Intraocular foreign bodies (IOFB) can cause ocular damage by various mechanisms. Pathology of eye trauma is of immense interest as lot of changes in the eye and adnexa are unique and are different from the pathology of rest of the body. This review was done to study pathological significance of ophthalmic FB in a referral tertiary institute of north-east India. Sixteen pathological specimens with various FBs were recorded. This study has been done for the first time in this part of the country.
\end{abstract}

Keywords: foreign body, enucleation, iron, copper, vegetative
Volume 7 Issue $3-2017$

\author{
Dipankar Das, Diva Kant Misra, Harsha \\ Bhattacharjee, Deepika Kapoor, Hemalata \\ Deka, Jayanta Kumar Das, Ganesh Chandra \\ Kuri, Panna Deka ,Apurba Deka \\ Sri Sankaradeva Nethralaya, India
}

\begin{abstract}
Correspondence: Dipankar Das, Senior Consultant, Department of Ocular Pathology, Uveitis \& NeuroOphthalmology Services, Sri Sankaradeva Nethralaya, Beltola, Guwahati - 781028, Assam, India, Tel 0361-2228879,

Fax 0361-2228878,Email dr_dasdipankar@yahoo.com
\end{abstract}

Received: July 26, 2017 | Published: August 28, 2017

\section{Introduction}

Foreign bodies (FB) in eye and adnexa are important in ophthalmology particularly in traumatic cases. ${ }^{1,2}$ A FB must possess sufficient momentum and energy to travel and penetrate the eye along with its deeper structures. ${ }^{1-3}$ Therefore, most posterior segment FBs is derived from metal fragments. A vegetative FB such as wood may reach inner part of eye but is located usually in the anterior segment. ${ }^{3,4}$ Animal matter such as cilia can be carried along with denser matter to the posterior segment. .,2,5 $^{12}$ Ocular damage caused by intraocular foreign bodies (IOFB) can occur by two mechanisms; (1) penetrating injury with its secondary complications and (2) by the presence of IOFB itself causing damage from microbial endophthalmitis, toxicity and other inflammatory reactions. ${ }^{4,6,7}$ Pathological evaluation of various FB in the eye and adnexa can give us bunch of information about the damages to the structures.

Aim: To study pathological significance of ophthalmic FB in a referral tertiary institute of north-east India.

\section{Materials and methods}

This is a hospital based, retrospective, laboratory study conducted in a tertiary ophthalmic institute of north-east India between 2010 to 2015. Different tissues including eyeball specimens received in ocular pathology laboratory were studied in details. Gross examinations were carried out in the (LEICA S6D) dissection microscope and were documented. Histopathological analysis was carried out with Hematoxylin and Eosin (H\&E) stain, other special stains and polarizer were used in (ZEISS) microscope Axioskop 40 and documented in (AxioCamMRc) camera. Specialized stains included Perl's stain for iron body, tissue Grams's stain (GIS) for microorganisms, Grocott's methenamine silver stain (GMS) for fungus, periodic acid schiff stain (PAS) for fungus and basement membrane of different structures, Congo red for amyloid to see ancillary pathologies. Sixteen pathological specimens with various FBs were seen and classified. Further hospital records were reviewed for the clinical profile, and other ancillary investigations to correlate the findings.

\section{Results}

Six cases of iron FB s were seen and acknowledged. One case of copper FB was seen in intraocular specimen with phthisical blind eye which was confirmed by scanning electron microscopy (SEM) (Figure 1a) and Energy dispersive X-ray spectroscopy (EDX) (Figure 1b). Three cases of vegetative FB were seen in the orbital/ lid tissues (Figure 2). FBs were detected in pathological specimen of two sympathetic ophthalmia cases (Figure 3). Urate crystals were documented in one of the conjunctival specimen (Figure 4a). One case of tripod glass wool FB (Mercedes Benz sign) was documented in a conjunctival specimen (Figure 4b). A case of insect body in the tissue (Figures $4 \mathrm{c}, \mathrm{d}, \mathrm{e}$ ) and other case of cilia was detected in the cohort. All iron FB were identified and measured. Out of six iron FBs, four had positivity with Perl's stain in the tissue sections. One iron FB had changes in the lens and its surrounding in a phthisical enucleated eyeball. One out of the three cases of vegetative FB had associated fungal infection (Aspergillus). Results of all FBs are shown in Figure 5 .

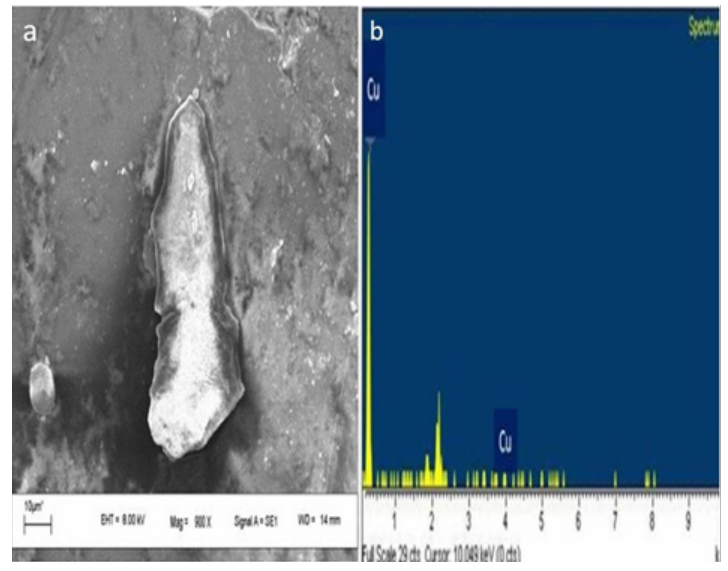

Figure I (a) Showed SEM picture of retrieved copper FB and (b) showed the EDX graph of same copper isolation in spectroscopy. 


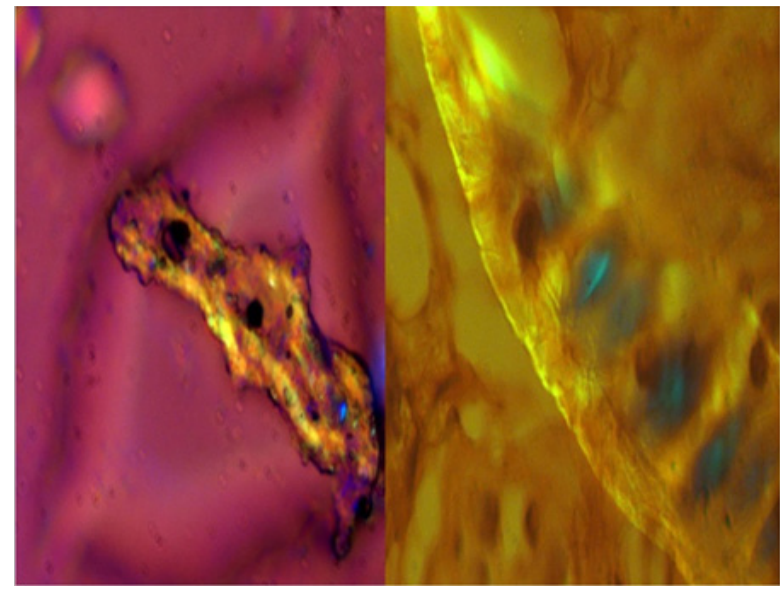

Figure 2 Showed vegetative $\mathrm{FBs}$ under polarizer in Zeiss microscope Axioskop 40 and captured in AxioCamMRc camera.

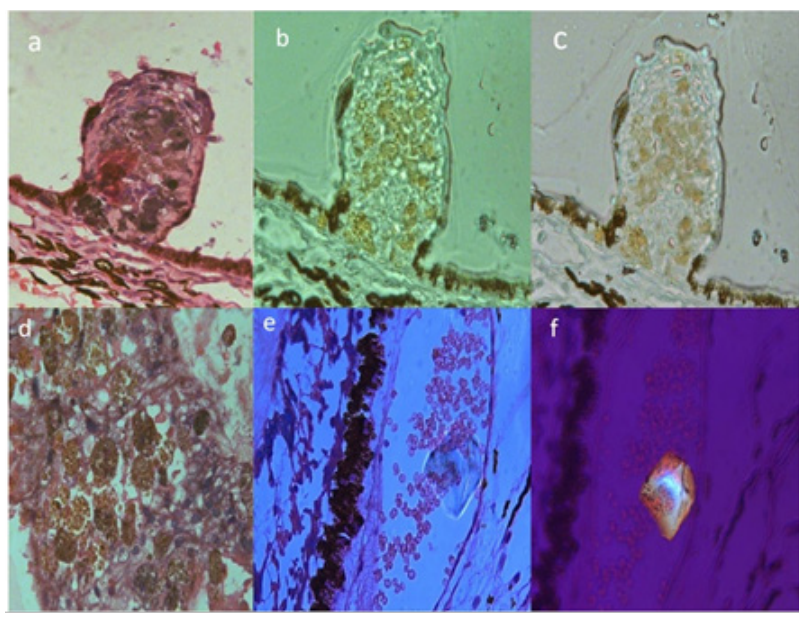

Figure 3 (a) Showed the case of sympathetic ophthalmia with Dalen Fuchs nodule in H\&E stain ( $X 400)$; (b, c) IHC for epitheloid cells marker positive $(X$ 400); (d) showed macrophages with inflammatory cells in H\&E stain (X 200) and $(e, f)$ showed refractile $F B$ in the choroid in the same eye.

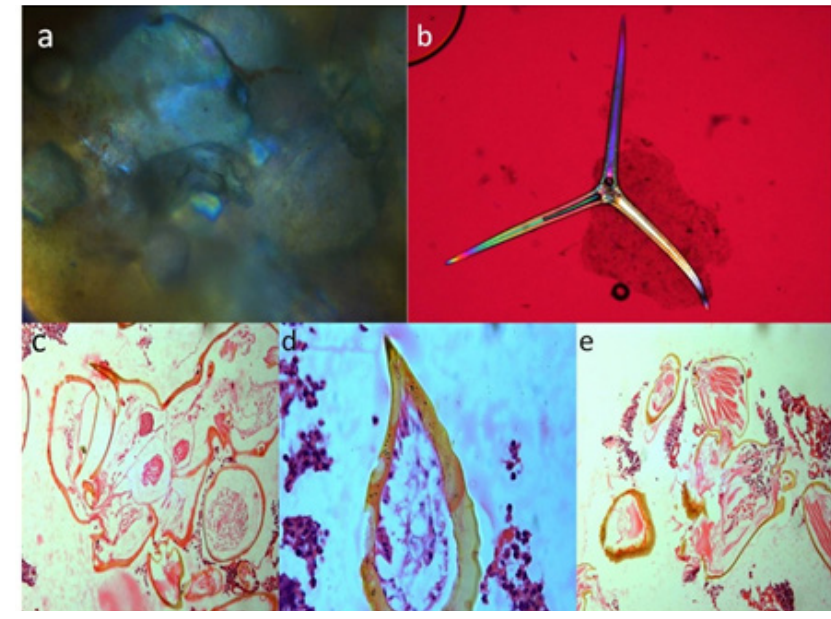

Figure 4 (a) Showed urate crystals deposit under polarizer; (b) showed tripod glass wool FB (Mercedes Benz sign) under polarization; ( c, d, e) showed insect bodies in the tissue (H\&E, X 200).

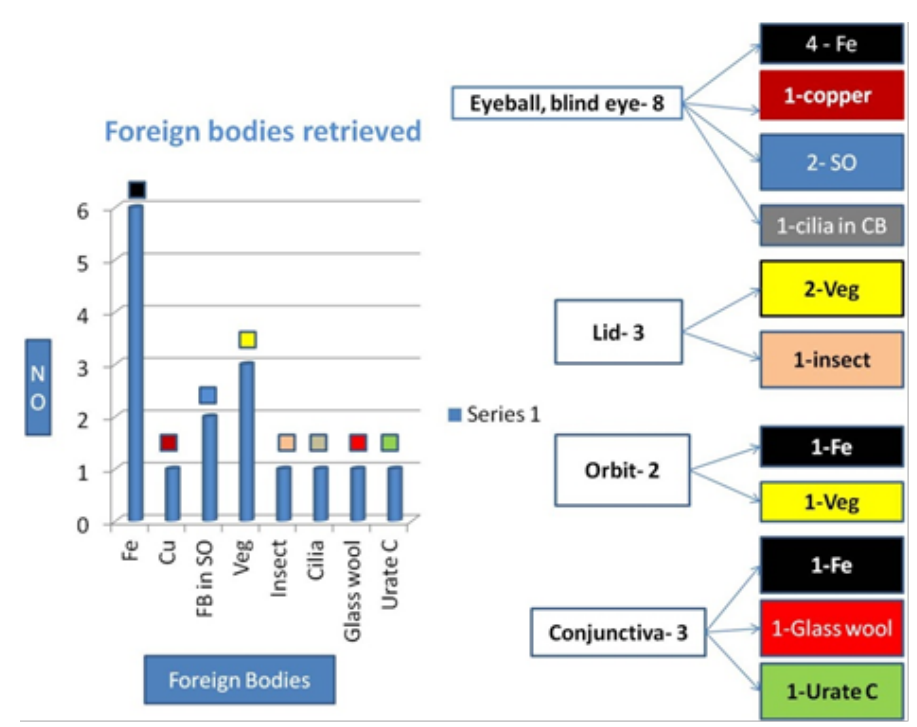

Figure 5 Showed bar diagram of various FBs retrieved in the study.

\section{Discussion}

Management of eye trauma is of concern to both the beginner ophthalmologists as well as tertiary eye care specialists. ${ }^{1-4}$ Pathology of eye trauma is of immense interest as many of the changes in the eye and adnexa are different from the pathology of other part of the body. ${ }^{3,4}, 7$ An understanding of the nature of activity of different FBs are important for medico legal purposes and in determining its nature, need for urgent removal. ${ }^{6,7} \mathrm{FBs}$ in cornea, anterior chamber, iris, angle of anterior chamber, lens, ciliary body, vitreous, retina, choroid, optic nerve etc have a far reaching impact on visual status and function of the eye. In our series, most of the iron FBs which had larger size caused significant damage to the structures, consistent with other studies. $3,4,6,7$ One of the iron FB lodged in crystalline lens caused microscopic changes in the lens capsules and the adjoining areas. In two cases, retinal pigment epithelium (RPE) showed degenerative changes with iron FB. Copper FB was seen and documented in SEM and EDX .Pure copper usually produces a suppurative reaction and alloys of $80 \%$ copper or less may subsequently lead to chalcosis. ${ }^{6}$ Zinc and aluminum IOFBs are toxic and should be removed immediately. One of the vegetative FB showed adjoining Aspergillus fungal infection in the tissue which has also been reported in the literature. ${ }^{6,8}$ It is always urgent and mandatory to remove the vegetable IOFBs because they usually contain microorganisms that can lead to endophthalmitis and cause excessive inflammation. ${ }^{6,8}$

Glass and plastic IOFBs are often well tolerated; they may be retained indefinitely in many cases if there is no surrounding damage and if it is immobile. ${ }^{4,6,8}$ In some cases, stone fragments may be well tolerated but the risk of seeding with micro-organisms merits their prompt removal. ${ }^{4,5}$ Sympathetic ophthalmia is characterized by granulomatous pan-uveitis following penetrating injuries of the globe which can be accidental or surgical. Cases of sympathetic ophthalmia show an increasing trend in our population. ${ }^{9}$ Classically in pathological samples, the uveal tract is thickened by diffuse lymphocytic infiltrate containing epitheloid cells and giant cells containing phagocytosed pigments. Eosinophils may be present specially in early cases and tend to collect beneath Bruch's membrane. ${ }^{6,8}$ Plasma cells are usually absent and in most of the cases choriocapillaris and retina are not 
involved in the inflammatory process. Dalen Fuchs nodules composed of transformed RPE cells or histiocytes that gain access to this location through the breaks in Bruch's membrane. Immunohistochemistry (IHC) studies indicate that Dalen Fuchs nodules consist of mixture of monocyte derived cells and hyperplastic RP cells. In our case series, we had two atypical histopathological patterns in the form of FBs at the level of the choroid and RPE in the enucleated exciting phthisical eye.

Sub-conjunctival urate crystals were seen in one non traumatic eye of elderly patient reported in the institute for cataract surgery. ${ }^{10}$ Interesting isolated case of insect bodies, glass wool tripod FB and cilia were seen. These were previously reported in various literatures.,11 Iron particles following use hammer and chisel comprises of almost $50 \%$ of the intra ocular foreign bodies isolated, which is consistent with our study. ${ }^{12}$ The severity of intraocular damage caused by IOFBs is decided by several factors. Wound length is predictive of the risk of retinal injury. A small external wound is suggestive of retinal damage as less of the IOFBs energy is dissipated during penetration, allowing it to reach and injure the retina. Foreign bodies entering the eye through sclera are more damaging than the corneal wound of entry. Retained IOFBs exhibit long-term toxicities like siderosis and chalcosis which may lead to retinal pigmentary changes and retinal toxicities as seen in our pathological samples. ${ }^{13}$ Endopthalmitisis following IOFB is most likely to occur if the foreign body is vegetative in nature. Work-related injuries and metallic IOFB are more prevalent in developed countries. ${ }^{14}$ This important pathological documentation of FBs has been done for the first time in this part of the country and its significance will help us to know various effects of FBs on the ocular tissues.

\section{Acknowledgments}

Sri Kanchi Sankara Health and Educational Foundation, Guwahati, India, IIT, Guwahati, India.

\section{Conflicts of interest}

There are no conflicts of interest.

\section{Funding}

None.

\section{References}

1. Smith VH. The incidence of intraocular foreign bodies. Int Ophthalmol Clin. 1968;8(1):137-146.

2. Percival SP. A decade of intraocular foreign bodies. Br J Ophthalmol. 1972;56(6):454-461.

3. Coleman DJ, Lucas BC, Rondeau MJ, et al. Management of intraocular foreign bodies. Ophthalmology. 1987;94:1647-1673.

4. Barry DR. Effects of retained intraocular foreign bodies. Int Ophthalmol Clin. 1968;8:153-170.

5. Gupta AK, Ghosh B, Majumdar S, et al. An unusual intraocular foreign body. Acta Ophthalmol Scand. 1996;74(2):200-201.

6. Rosenthal AR, Marmor MF, Leuenberger P, et al. Chalcosis: A study of natural history. Ophthalmology. 1979;86(11):1956-1972.

7. Tawara A. Transformation and cytotoxicity of iron in siderosis bulbi. Invest Ophthalmol Vis Sci. 1986;27(2):226-236.

8. Spencer WH. Autoimmune diseases. In Spencer WH, (Edn.),: Ophthalmic pathology: an atlas and textbook, WB Saunders, USA: Philadelphia; 1986. p. 1913-1956.

9. Das D, Bhattacharjee H, Das $\mathrm{K}$, et al. The changing patterns of uveitis in a tertiary institute of Northeast India. Indian J Ophthalmol. 2015;63(9):735-737.

10. Sharma P, Das D, Deka P, et al. Subconjunctival urate crystals. Cornea. 2010;29(7):830-832.

11. Babu K, Marelihelli RE. Insect wing tarsal foreign body causing conjunctival granuloma and marginal keratitis. Indian J Ophthalmol. 2009;57(6):473-474.

12. Singh R, Kumar A, Gupta V, et al. 25-Gauge active aspiration silicon tip-assisted removal of glass and other intraocular foreign bodies. Can J Ophthalmol. 2016;51(2):97-101.

13. Loporchio D, Mukkamala L, Gorukanti K, et al. Intraocular Foreign Bodies: A Review. J survophthal. 2016;61(5):582-596.

14. Liu CC, Tong JM, Li PS, et al. Epidemiology and clinical outcome of intraocular foreign bodies in Hong Kong: a 13-year review. Int Ophthalmol. 2017;37(1):55-61. 